\title{
The lateral habenula interacts with the hypothalamo-pituitary adrenal axis response upon stressful cognitive demand in rats
}

\author{
Victor Mathis $^{\mathrm{a}, \mathrm{b}, 1}$, Brigitte Cosquer ${ }^{\mathrm{a}, \mathrm{b}}$, Alexandra Barbelivien ${ }^{\mathrm{a}, \mathrm{b}}$, Karine Herbeaux ${ }^{\mathrm{a}, \mathrm{b}}$, \\ Béatrice Bothorel $^{\mathrm{c}}$, Dominique Sage-Ciocca ${ }^{\mathrm{d}}$, Vincent-Joseph Poirel ${ }^{\mathrm{c}}$, Chantal Mathis ${ }^{\mathrm{a}, \mathrm{b}}$, \\ Lucas Lecourtier ${ }^{\mathrm{a}, \mathrm{b}, *}$ \\ ${ }^{\text {a }}$ Laboratoire de Neurosciences Cognitives et Adaptatives (LNCA), Université de Strasbourg, F-67000 Strasbourg, France \\ ${ }^{\mathrm{b}}$ LNCA, UMR 7364-CNRS, F-67000 Strasbourg, France \\ ${ }^{\mathrm{c}}$ Institut des Neurosciences Cellulaires et Intégratives (INCI), UPR-3212, CNRS, 5 Rue Blaise Pascal, 67084 Strasbourg, France \\ ${ }^{\mathrm{d}}$ Chronobiotron UMS 3415, 5 Rue Blaise Pascal, 67084 Strasbourg, France
}

\section{A R T I C L E I N F O}

\section{Keywords:}

Lateral habenula

Memory

Stress

Corticosterone

\begin{abstract}
A B S T R A C T
The lateral habenula ( $\mathrm{LHb}$ ) is involved in emotional and cognitive behaviors. Recently, we have shown in rats that blockade of excitatory inputs to the LHb not only induced deficits of memory retrieval in the water maze, but also altered swim strategies (i.e., induced excessive thigmotaxis). The latter observation, although consistent with the occurrence of memory deficits, could also possibly be the consequence of an excessive level of stress, further suggesting a role for the LHb in the stress response in our behavioral paradigm. To test this hypothesis we performed in rats intra-LHb infusion of 6-cyano-7-nitroquinoxaline-2,3-dione (CNQX, $267 \mathrm{ng} / \mathrm{side}$ in $0.3 \mu \mathrm{L}$ ), or vehicle, and assessed the responsiveness of the hypothalamo-pituitary adrenal (HPA) axis to environmental stressful or non-stressful situations. We have measured plasma corticosterone (CORT) concentrations at different time points before and following intra-LHb infusion of CNQX - or of the same volume of vehicle - in three conditions: during the probe test of a water maze experiment; in an anxiety test, the elevated plus maze; and in a home cage condition. Whereas there were no differences in the home cage condition and in the elevated plus maze, in the water maze experiment we observed that CNQX-treated rats presented, along with memory deficits, a higher level of blood CORT than vehicle-treated rats. These results suggest that perturbations of the modulation of the HPA axis are consecutive to the alteration of LHb function, whether it is the result of a defective direct control of the LHb over the HPA axis, or the consequence of memory deficits.
\end{abstract}

\section{Introduction}

The lateral habenula ( $\mathrm{LHb}$ ) plays an important role in emotional behaviors, including the coding of aversive events [1,2,3] and of negative motivational value [4], fear response [5,6], and anxiety-related behavior [7]. Recently, we have found in rats that alteration of LHb function impaired encoding and recall of spatial reference memory [8], as well as working memory [9], suggesting that the LHb was crucially involved in the online processing of sensory information necessary to adapt behavior. Interestingly, we have found in the water maze experiment that the blockade of excitatory inputs to the LHb, by means of intra-LHb administration of the AMPA/Kainate antagonist 6-cyano-7nitroquinoxaline-2,3-dione (CNQX), not only impaired retrieval of spatial reference memory, but also increased the time spent by the rats swimming along the edge of the water maze, a swim pattern referred to as "thigmotaxis" [8]. Such a behavior is considered as the "default" swim strategy, typically engaged by rats when they start being tested in the water maze and have not yet acquired knowledge of the presence of an escape platform [10]. The persistence of thigmotaxis, upon repeated testing, reflects not only the presence of memory deficits, but is also considered in rodents to be the consequence of a higher anxiety level $[11,12]$. These views suggest that the spatial memory deficits observed following intra-LHb CNQX administration could partly be due to a fault in stress adaptation of the animals, a particularly interesting issue considering the impact of stress on memory retrieval [13]. The possible involvement of the LHb in coping with stressful situations would not appear surprising as numerous studies have demonstrated that the LHb is recruited following different types of stressful experiences including restraint, tail pinch, exposure to a novel environment, or swimming $[14,15,16]$. The fact that LHb dysfunction induced memory deficits

\footnotetext{
* Corresponding author at: LNCA, UMR 7364, CNRS, Université de Strasbourg, 12 rue Goethe, F-67000 Strasbourg, France.

E-mail address: 1.lecourtier@unistra.fr (L. Lecourtier).

${ }^{1}$ Present address: Department of Neuroscience, Icahn School of Medicine at Mount Sinai, New York 10029-6574, USA.
} 
along with the marked thigmotaxis, suggesting behavioral maladaptation, is also consistent with early hypotheses by Thornton and colleagues $[17,18]$ who postulated that LHb dysfunction impairs decision making in stressful situations. Overall, these findings and hypotheses position the LHb at the interface between cognition and emotion, while it is well known that defective stress coping has detrimental effects on cognitive behaviors $[19,20]$. In rodents, the response to stress has extensively been shown to involve, among other physiological mechanisms, the activation of the hypothalamo- pituitary adrenal (HPA) axis and the subsequent release of corticosterone (CORT) in the blood [21]. The consequences of the CORT response on memory abilities have been shown to follow an inverted U-shaped curve with memory impairments occurring when CORT release is either too low or too high [20,22,23]. A possible interaction between the LHb and the HPA axis has previously been demonstrated $[24,25]$. Therefore, to challenge the possibility that the memory deficits observed following alteration of LHb function could partly be the consequence of a defective stress response, we assessed the responsiveness of the HPA axis by measuring blood CORT levels during the probe trial of the water maze experiment. In addition, we assessed blood CORT levels during the performance of the elevated plus maze, which represents a very stressful experience devoid of cognitive aspects, and in a home cage condition, which presents a minimal level of stress, as rats still need to be manipulated and maintained in order to perform the microinjection, and is devoid of cognitive aspects.

\section{Materials and methods}

\subsection{Animals and surgery}

70 male Long-Evans rats (270-300 g at the time of surgery; Centre d'Elevage R. Janvier, France) were used in compliance with the European Committee Council Directive. This project has been validated by an ethical committee (Ministère de l'éducation nationale, de l'enseignement supérieur et de la recherche; APAFIS 505). Rats were housed individually under a 12:12 h light-dark cycle (light on at 7:00 A.M) with ad libitum access to food and water. Rats were anesthetized by intraperitoneal injection of a mixture of ketamine $(82.5 \mathrm{mg} / \mathrm{kg})$ and xylazine $(11 \mathrm{mg} / \mathrm{kg})$ and placed within a stereotaxic frame (flat skull). Lidocaine $(0.1 \mathrm{ml})$ was subcutaneously injected at the incision site prior to surgery. Once the holes were drilled, stainless steel guide cannulae were gently bilaterally inserted $1 \mathrm{~mm}$ above the LHb at the following coordinates (in $\mathrm{mm}$ ): anteroposterior, -3.9 from Bregma; mediolateral, \pm 0.7 ; dorsoventral, -3.6 from dura. Guides, in which dummy cannulae were inserted, were secured to the skull and 3 screws with dental cement. Rats were administered an antibiotic (amoxicilline, intramuscular, $10 \mathrm{mg} / \mathrm{kg}$ ), a painkiller (meloxicam, subcutaneous, $2 \mathrm{mg}$ / $\mathrm{kg}$ ), and given a 10-day recovery period. Three different batches of rats were used, one for each experiment, i.e., home cage $(n=21)$, water maze $(n=29)$, and elevated plus maze $(n=20)$. The experiments took place during the first half of the light period, between 09:00 A.M. and 01:00 P.M., which is the period of the light-dark cycle when circulating CORT is at its lowest level [26].

\subsection{Water maze}

Spatial memory was assessed as previously described [8]. Training comprised one block of four consecutive trials (60 s max per trial) per day for 5 consecutive days, with the hidden platform situated at the same location throughout training. One day after completion of training, the platform was removed and a $60 \mathrm{~s}$ retention test was performed. During training all rats were administered vehicle within the LHb before each session. Following training, treatment groups were composed so that there was no statistical difference between their training performances for all parameters analyzed. The movements of the rats were followed by a computer-based video tracking system. Training analyses comprised, for each trial, the distance to reach the platform, the mean swim speed, the resting time, i.e., when rats are floating, and the percentage of time spent in thigmotaxis, i.e., when rats are swimming along the edge of the water maze within a $10 \mathrm{~cm}$-wide corridor. Retention test analyses comprised the time spent within the target quadrant - i.e., the quadrant where the platform was located during training -, the number of crossings of the platform location, the mean distance from the platform location, the percentage of time spent in thigmotaxis, the total distance swum, the mean swim speed, and the resting time.

\subsection{Elevated plus maze}

Anxiety was assessed in the elevated plus maze (EPM) test. The EPM, made of black Plexiglas, was elevated $73 \mathrm{~cm}$ above the floor and consisted of four arms $(50 \mathrm{~cm} \times 10 \mathrm{~cm})$, two comprising $40 \mathrm{~cm}$-high walls (closed) and two comprising $1.5 \mathrm{~cm}$-high borders (open). Light intensity was set at 7.5 lux in the open arms, 5 lux at the center of the maze, and 2 lux in the closed arms. The test lasted $5 \mathrm{~min}$ and the number of entries and time spent in each arm, were scored. The maze was cleaned with water and absolute ethanol between each rat.

\subsection{Drug and infusion procedure}

Once the dummy cannulae were taken out, infusion cannulae were bilaterally inserted, protruding $1 \mathrm{~mm}$ below the guides, and $0.3 \mu \mathrm{l}$ of a $0.89 \mu \mathrm{g} / \mu \mathrm{l} \mathrm{CNQX}$ solution - or the same volume of vehicle [2\% DMSO/ aCSF: (in $\mathrm{mM}$ ) NaCl, $\left.145 ; \mathrm{KCl}, 2.7 ; \mathrm{MgCl}_{2}, 1.0 ; \mathrm{CaCl}_{2}, 1.2\right]$ - was bilaterally administered over $1 \mathrm{~min}$. Infusion cannulae were left in place for an additional $30 \mathrm{~s}$ before being removed and the dummy cannulae reinserted. In both the EPM and the water maze experiments testing was performed $15 \mathrm{~min}$ following the administration of the treatments.

\subsection{Measurements of plasma CORT}

The blood sampling method was inspired by Flutter et al. [27]. After rats recovered from surgery, they were gently handled a few minutes per day for one week in order to minimize handling stress effect on the day when blood sampling was performed. To this aim, the experimenter placed the rat under a towel to minimize the animal movements and to easily expose the tail for subsequent blood sampling. The water maze experiment comprised 5 days of training, without blood sampling, followed by a probe trial on day 6 , before and following which blood samples were collected (see Fig. 1); in order to habituate rats to such blood sampling, and therefore reduce stress, rats underwent the handling procedure used to perform blood sampling on each of the 5 days of training, once before and once following each session. In the home cage condition, blood sampling was performed 5 days after the litter was changed in order to avoid possible disturbances due to the resulting change in housing environment, which may lead to an increased level of circulating CORT. On the day of testing in each experiment, a second experimenter - to the presence of whom the rats were also habituated gently performed a small incision of the rat's tail to collect blood samples. The incision was performed with a razor blade at the level of the dorsal vein. Blood was directly collected in heparin-coated capillary tubes. As blood sampling was performed several times (twice in the water maze and elevated plus maze tests and 3 times in the home cage condition; Fig. 1), the first incision was performed on the caudal third of the tail, and subsequent incisions performed at progressively rostral intervals of approximately $5 \mathrm{~mm}$, in order to avoid a reduction in collected blood volume due to coagulation. The volume of blood samples ranged between 200 and $300 \mu \mathrm{L}$. Following blood sampling the tail was cleaned and dried with a tissue and the rat gently replaced in its home cage. The different timings of blood sampling in all testing conditions are summarized in Fig. 1. For all conditions, at least a 90 min delay was introduced between the first blood sampling (i.e., baseline) and the subsequent manipulations in order to allow rats to recover from it; such 
A

WATER MAZE

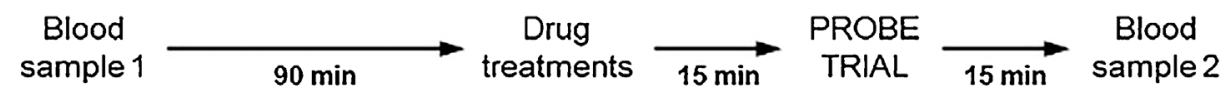

O Vehicle (11) O CNQX (10)

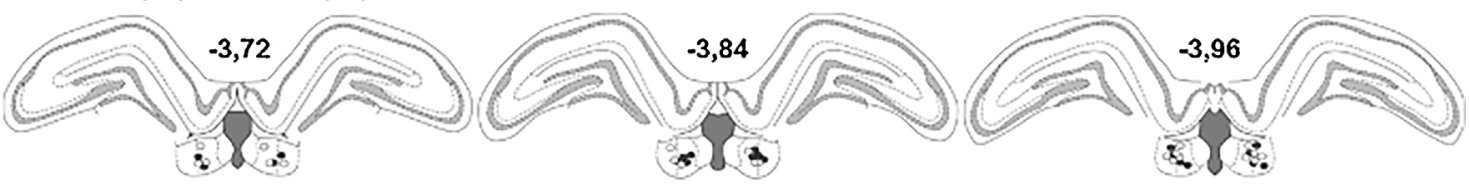

B

ELEVATED PLUS MAZE

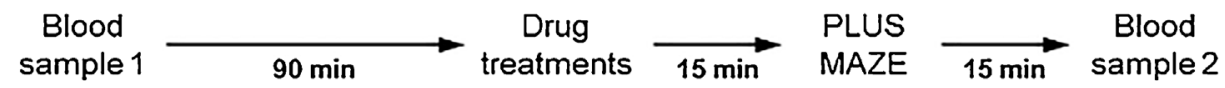

$\circ$ Vehicle (9) O CNQX (11)

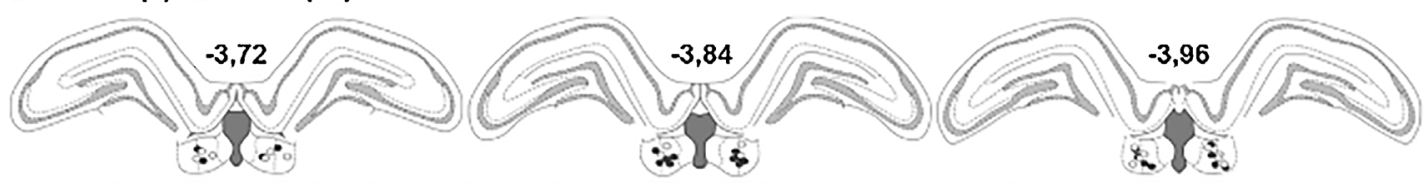

C HOME CAGE

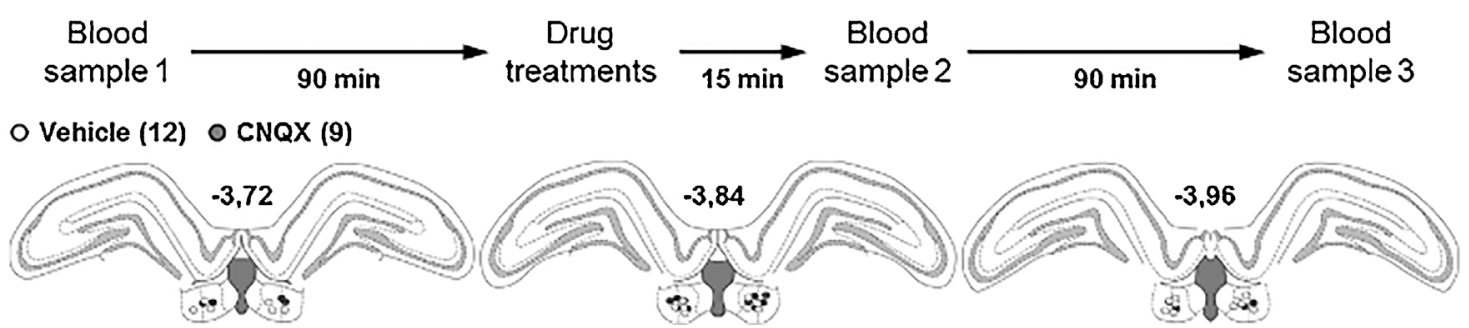

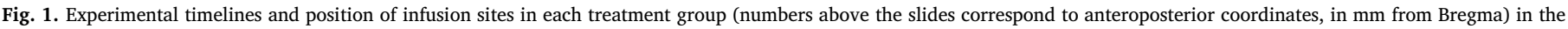
water maze (A), elevated plus maze (B), and home cage (C) experiments.

a delay has been found to be sufficient to allow blood CORT levels to return to baseline following an acute stress [28]. In the water maze experiment, the second blood sampling was performed $15 \mathrm{~min}$ following the performance of the retention test in order to assess the peak of CORT release which typically occurs $15 \mathrm{~min}$ after the beginning of a swimming exercise [29]. The same timing was used for the EPM experiment, where the second blood sample was collected $15 \mathrm{~min}$ after rats performed the task. All blood samples were centrifuged $(3000 \mathrm{rpm}$, at $4{ }^{\circ} \mathrm{C}$ ). The plasma was subsequently collected and stored at $-20{ }^{\circ} \mathrm{C}$ until the performance of the assay. Circulating CORT concentrations were assessed in duplicates using a commercially available RIA kit (MP Biomedical, Loughborough, UK). The sensitivity limits of the assay were comprised between $7.7 \mathrm{ng} / \mathrm{ml}$ and $1000 \mathrm{ng} / \mathrm{mL}$. The reproducibility of the method was determined by evaluating intra-assay variation $(7.1 \%)$ and inter-assay variation (6.5\%).

\subsection{Histological procedures}

Following testing rats underwent a bilateral microinfusion of a $0.1 \%$ Evans blue-CNQX solution $(0.89 \mu \mathrm{g} / \mu \mathrm{l}, 0.3 \mu \mathrm{L} /$ side $)$ at the injection sites. Rats were subsequently deeply anesthetized with pentobarbital $(100-120 \mathrm{mg} / \mathrm{kg}$, ip). Following intracardiac perfusion of phosphatebuffered saline (PBS, $0.1 \mathrm{M}$ ) and then $4 \%$ paraformaldehyde (PFA)-PBS solution ( $\mathrm{pH} 7.4 ; 4{ }^{\circ} \mathrm{C}$ ), brains were removed and post-fixed in $4 \%$ PFAPBS $\left(4^{\circ} \mathrm{C}, 48 \mathrm{~h}\right)$. Brains were then transferred into a $0.1 \mathrm{M}$ PBS- $20 \%$ sucrose solution $\left(4^{\circ} \mathrm{C}, 48 \mathrm{~h}\right)$ and subsequently frozen. Serial $40 \mu \mathrm{m}$ thick sections were cut in the coronal plane at $-22^{\circ} \mathrm{C}$ and collected on gelatin-coated slides. As previously described [9], the red fluorescence emitted by the Evans blue was observed under a microscope (excitation at $620 \mathrm{~nm}$, emission at $680 \mathrm{~nm}$ ); out of the 70 operated rats, 62 had fluorescence restricted to the LHb and were kept for the analyses of the consequences of CNQX administration into the LHb, groups being composed as follows: water maze experiment (vehicle, $\mathrm{n}=11$; CNQX, $\mathrm{n}=10$ ); elevated plus maze (vehicle, $\mathrm{n}=9$; $\mathrm{CNQX}, \mathrm{n}=11$ ); home cage experiment (vehicle, $\mathrm{n}=12$; CNQX, $\mathrm{n}=9$ ) (Fig. 1). In addition, in the water maze experiment, 8 rats had fluorescence almost exclusively within the thalamic region situated ventrally to the LHb, indicative of the misplacement of the guide cannulae and therefore of infusion sites; those rats were included in a "thalamus" control group (vehicle, $n=4$; CNQX, $n=4$ ). In each rat of the present study, no fluorescence was detected within the hippocampal region situated dorsally to the LHb.

\subsection{Statistical analyses}

In the figures, data are represented as mean \pm SEM and group sizes are indicated between brackets next to the treatments. In addition, individual performances are represented by white circles; because of their partial or complete overlap, their number may appear smaller than the total number of rats. For the water maze experiment, treatment groups for the probe trial - vehicle vs CNQX - were composed according to training performances so that there was no difference in all variables analyzed (two-way ANOVAs with treatment as the between- subject factor and training day as the repeated measure) (see Supplementary material). Water maze retention test performances were analyzed with one-way ANOVAs with treatment as the between-subject factor; for the percentage of time spent in the target quadrant, average performance of each group was compared with chance level (25\%) using a $t$-test. Performances in the EPM (number of visits and percentage of time spent 


\section{$\square$ Vehicle (11) $\square$ CNQX (10)}

A

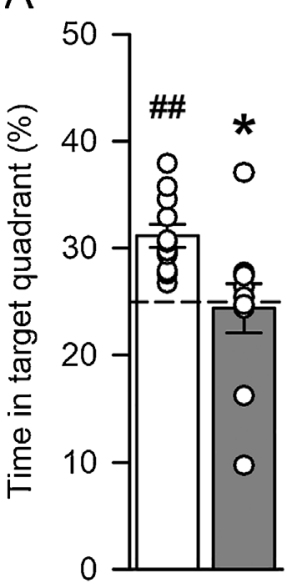

$\mathrm{B}$

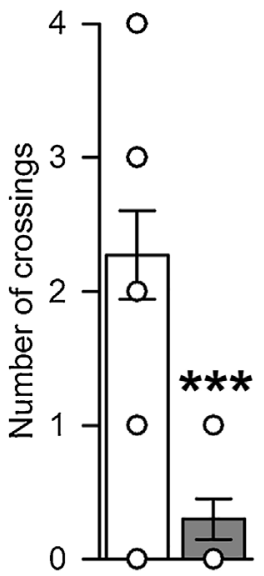

C

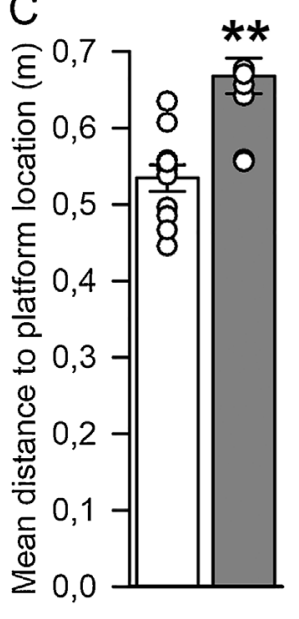

D

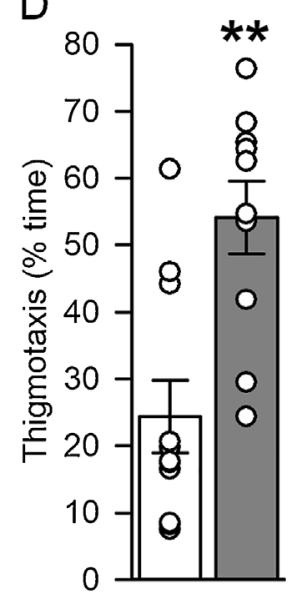

Fig. 2. Performance during the retention test of the water maze experiment. Retrieval deficits were visible in CNQXtreated rats which performed at chance (dashed line in A), made fewer crossings over the platform location (B), and swam significantly further from the platform location (C). In addition, CNQX increased the time spent in thigmotaxis (D) but did not affect the total distance swum (E), the mean swim speed (F) and the resting time (G). " $p<0.05,{ }^{* *} p<0.01$, ${ }^{* * * * *} p<0.001$ vs vehicle and ${ }^{\# \#} p<0.01$ vs chance.

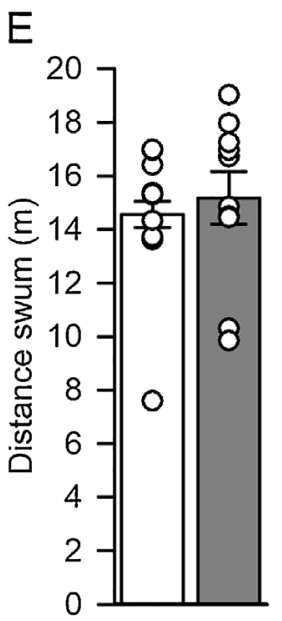

$\mathrm{F}$

\section{G}
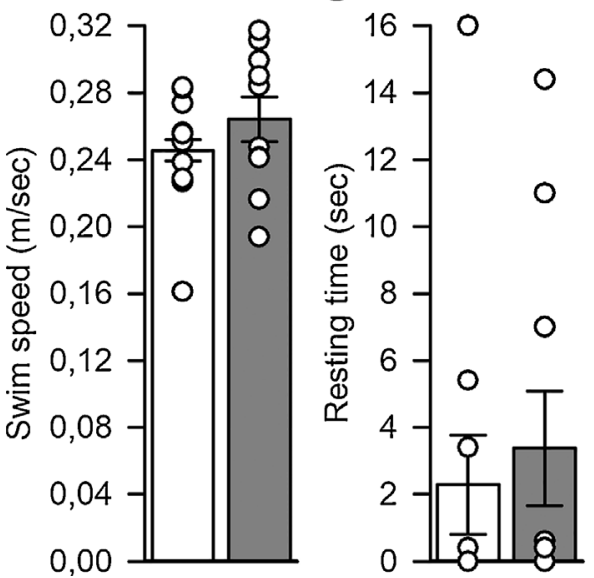

in the open and closed arms) were separately analyzed using $t$-tests. Analyses of the amount of plasma CORT were performed with ANOVAs with treatment as the between-subject factor and sample as the repeated measure (3 levels, home cage; two levels, water maze and EPM). Post hoc analyses used the Newman-Keuls multiple range test when appropriate. Values of $\mathrm{p}<0.05$ were considered significant.

\section{Results}

\subsection{Water maze probe trial}

Results are presented in Fig. 2. To assess memory we analyzed the time spent in the target quadrant and found a significant effect of group $\left(F_{1,19}=7.69, p<0.05\right.$; Fig. $\left.2 \mathrm{~A}\right)$; in addition, comparison of performances to chance level revealed that whereas vehicle-treated rats spent a significantly greater amount of time within the target quadrant $\left(t_{10}=5.69, p<0.001\right)$, CNQX-treated rats did not $\left(t_{9}=-0.27\right.$, $p>0.7$ ), suggesting that only control rats remembered the platform location. This result was confirmed by the analysis of the number of crossings which also indicated a significant effect of group $\left(F_{1,19}=27.12, p<0.0001\right)$, CNQX-treated rats making less crossings than vehicle-treated rats (Fig. 2B), and of the distance from the platform location $\left(F_{1,19}=21.74, p<0.001\right.$; Fig. $\left.2 \mathrm{C}\right)$. In addition, there was a significant difference in terms of the time spent in thigmotaxis $\left(F_{1,19}=15.01, p<0.01\right)$, CNQX-treated rats swimming more along the edge of the pool (Fig. 2D). Analyses of the other variables did not indicate significant differences [distance swum: $F_{1,19}=0.27, p>0.6$ (Fig. 2E); swim speed: $F_{1,19}=1.11, p>0.3$ (Fig. 2F); resting time:
$F_{1,19}=0.23, p>0.6$ (Fig. 2G)]. Altogether these results confirm that although intra-LHb CNQX administration induces memory deficits, it preserves motor abilities [8].

\subsection{Elevated plus maze}

Results are presented in Fig. 3. For the number of entries, as a measure of general locomotion, there were no significant differences in open $\left(t_{18}=-0.49, p>0.6\right)$ or closed $\left(t_{18}=-1.26, p>0.2\right)$ arms (Fig. 3A). For the percentage of time spent in arms, as a measure of anxiety, there was no effect of treatment in open $\left(t_{18}=-0.52\right.$, $p>0.6)$ or closed $\left(t_{18}=0.9, p>0.3\right)$ arms (Fig. $3 \mathrm{~B}$ ). The present results confirm that intra-LHb CNQX administration does not alter the level of anxiety of the rats [8].

\subsection{Measurements of plasma CORT}

For the water maze experiment (Fig. 4A), there was a significant effect of treatment $\left(F_{1,19}=6.16, p<0.05\right)$, a significant effect of sample $\left(F_{1,19}=272.38, p<0.0001\right)$ and a significant interaction between the two factors $\left(F_{1,19}=9.99, p<0.01\right)$. Post hoc analysis revealed that overall CORT levels were in both groups higher in sample 2 than in sample $1(p<0.001)$; moreover, whereas CORT levels of both groups did not differ in sample $1(p>0.7)$, in sample 2 they were higher in CNQX-treated rats $(p<0.001)$. For the EPM experiment (Fig. 4B), there was no significant effect of treatment $\left(F_{1,18}=0.41\right.$, $p>0.5)$, a significant effect of sample $\left(F_{1,18}=397.8, p<0.0001\right)$ and no significant interaction $\left(F_{1,18}=0.37, p>0.5\right)$; post hoc analysis 

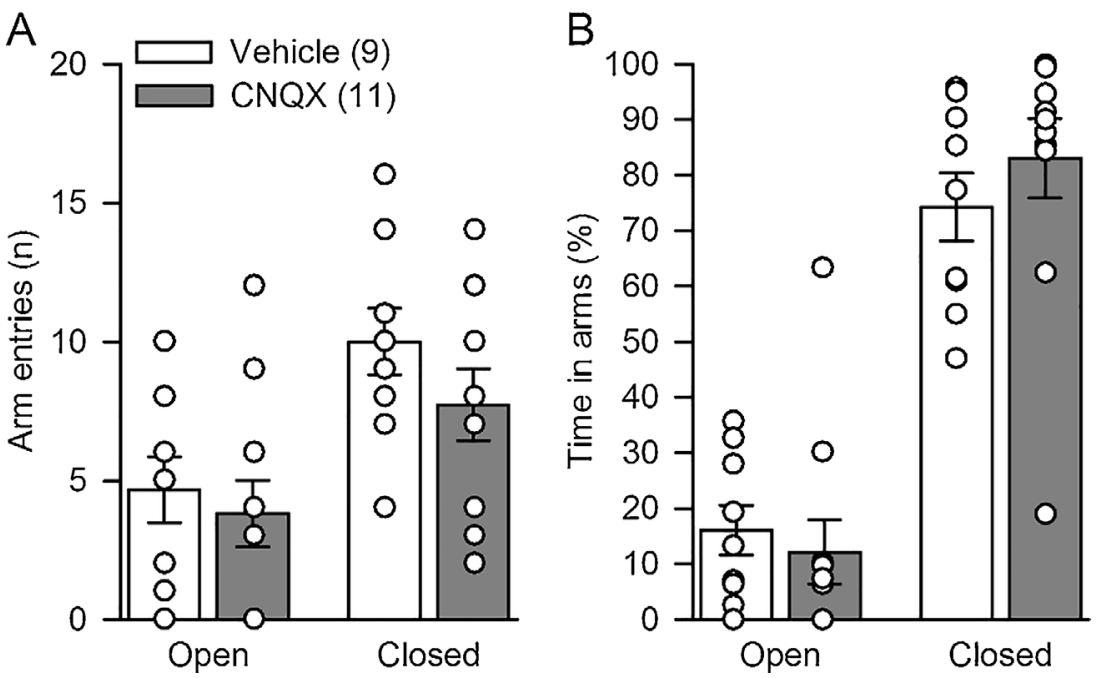

Fig. 3. Performance during the elevated plus maze test. There was no effect of CNQX administration on the behavior of the rats, as both groups made an equivalent number of visits to (A) and spent an equivalent time in (B) open and closed of arms. revealed that CORT levels were in both groups higher in sample 2 than in sample 1 ( $p<0.001)$. For the home cage experiment (Fig. 4C), there was no significant effect of treatment $\left(F_{1,19}=1.80, p>0.1\right)$, a significant effect of sample $\left(F_{2,38}=74.88, p<0.0001\right)$, and post hoc analysis revealed that CORT levels were in both groups significantly higher in sample 2 than in sample $1(p<0.001)$ and in sample 3 $(p<0.001)$, and that levels in sample 1 and 3 were not statistically different $(p>0.1)$; there was no significant interaction $\left(F_{2,38}=0.96\right.$, $p>0.3$ ), indicating that in both groups CORT levels fluctuated similarly over the course of the experiment. Altogether these results indicate that following intra-LHb CNQX administration the activity of the HPA axis was only altered during the water maze experiment; as such a disturbance was visible neither during the EPM experiment nor in the home cage condition. These results further suggest that the modulation of the HPA axis by the LHb preferentially occurs upon the concomitant presence of stress and cognitive demand.

3.4. Memory performances and CORT levels of animals with injection sites located within the thalamic region situated ventrally to the $\mathrm{LHb}$

Results are presented in Fig. 5. For the distance swum during training there was no difference between the groups $\left(F_{1,6}=0.006\right.$, $p>0.9)$, a significant effect of training day $\left(F_{4,24}=2.95, p<0.05\right)$ and no significant interaction between the two factors $\left(\mathrm{F}_{4,24}=1.87\right.$, $\mathrm{p}>0.1$ ). During the probe trial, analysis of the time spent in the target quadrant indicated no difference between the groups $\left(F_{1,6}=0.24\right.$, $p>0.6)$ while they both had performances significantly above chance level (Veh: $t_{3}=4.25, p<0.05$; CNQX: $t_{3}=3.37, p<0.05$ ). Analyses of all other training and probe trial variables indicated no effect of treatment, confirming previous findings [8]. According to CORT levels, there was no significant effect of treatment $\left(F_{1,6}=0.02, p>0.8\right)$, a significant effect of sample $\left(F_{1,6}=201.24, p<0.0001\right)$ and no significant interaction $\left(F_{1,6}=0.07, p>0.7\right)$.

\section{Discussion}

In the present study we showed in the water maze that blockade of excitatory glutamatergic inputs to the LHb prior to a probe trial impaired retrieval of spatial reference memory, confirming results of a previous study [8]. Such impairments were accompanied by higher plasma CORT levels. The effects observed are unlikely to result from an alteration of the function of the medial habenula $(\mathrm{MHb})$ or of this of the thalamic region underlying the LHb, through diffusion of the CNQX. Cullinan et al. [15] have found that following a swim exercise no c-Fos labeling was observed within the MHb, in contrast to a strong expression within the LHb, suggesting that the MHb is not particularly involved in the physiological response during swim paradigms. Injection of CNQX into the underlying thalamic region had no effect on memory
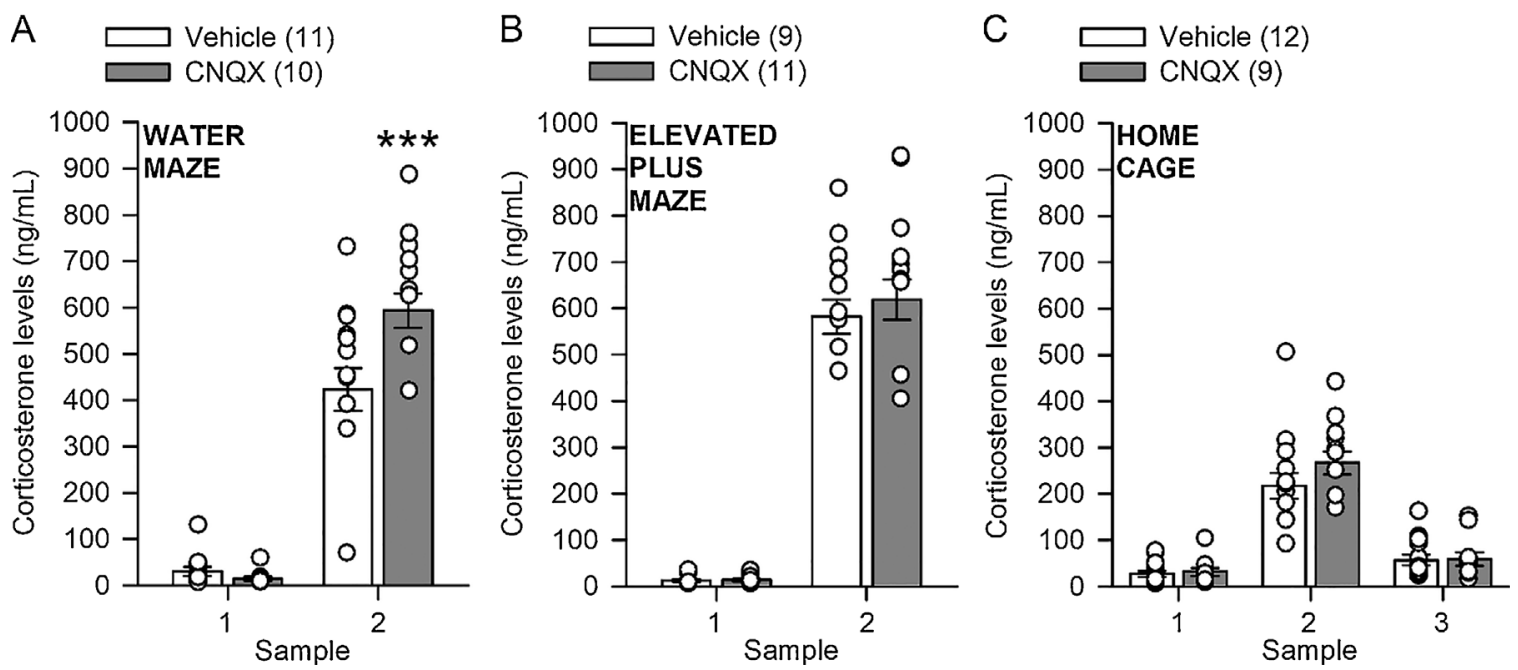

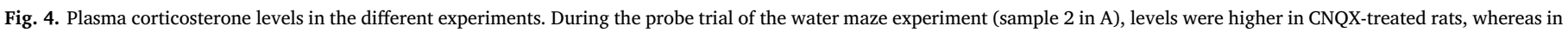
the elevated plus maze (B) and in the home cage (C) experiments there was no difference between groups. ${ }^{* * * k}<0.0001$ vs vehicle at the same time-point. 
A

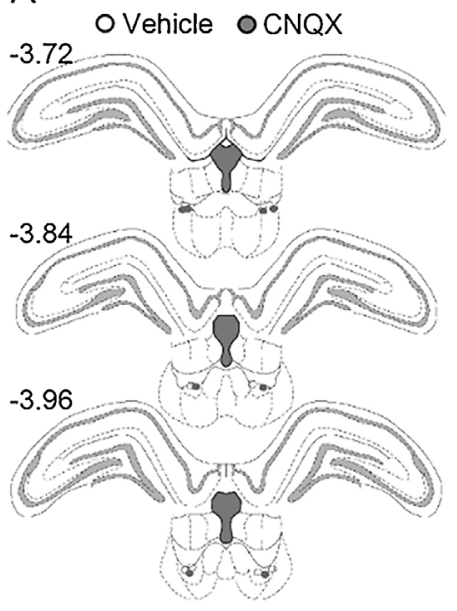

B

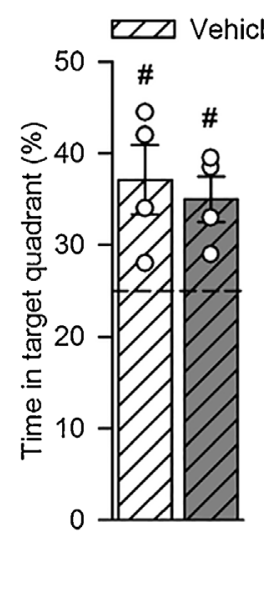

C

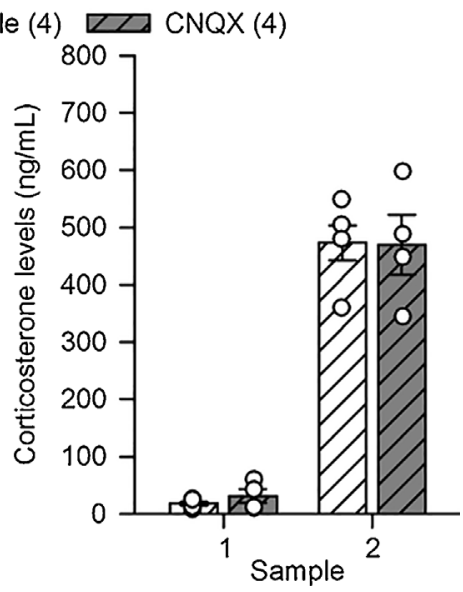

Fig. 5. Position of infusion sites (A), retrieval performance (B) and corticosterone levels (C) during the probe trial of the water maze experiment of rats which were administered treatments within the thalamic region underlying the LHb. CNQX impacted neither retrieval performance nor corticosterone levels. ${ }^{\#} p<0.05$ vs chance. performance or CORT release (Fig. 5). Altogether, these data strengthen the view that the observed deficits are likely to result solely from an alteration of LHb activity. Increased CORT release is mainly associated with the response of the HPA axis to stress; however, CORT release also follows a circadian rhythm, participates in the anticipation of behavioral activation during rodents' active phase, and is also stimulated in situations of intense behavioral activation [30]. One could therefore consider that the CNQX-induced increased CORT release observed in the water maze experiment only reflects the increase of rats' activity. However, this hypothesis seems rather unlikely as in the water maze experiment there was no difference between CNQX- and vehicle-treated rats in terms of the distance swum, the swim speed and the resting time (Fig. 2), thus suggesting similar levels of behavioral activity. In addition, this lack of difference in behavioural activation rules out the presence of motor deficits, confirming the demonstration, using the beam walking test, that intra-LHb CNQX administration preserves sensorimotor coordination [8].

It is particularly striking that intra-LHb CNQX administration affected plasma CORT concentration only in the water maze. Although in the elevated plus maze a difference in CORT levels between both groups was not expected, as CNQX did not alter anxiety levels, a possible explanation for this is a possible ceiling effect, whereby the response of the HPA axis is maximum and plasma CORT levels could not be raised further in this condition. If this was the case, a difference could likely be detected in the home cage condition, which represents a stressful experience, but of certainly much lower intensity; however, in this situation there was no difference between CNQX-treated animals and controls although CORT levels were much lower than in the elevated plus maze. Therefore, it is unlikely that what was observed during the elevated plus maze experiment was the reflection of a ceiling effect, and in consequence the higher level of CORT observed in the water maze in CNQX-treated rats is likely to reflect a higher response of the HPA axis which is specific to this testing condition.

To explain the occurrence of higher CORT release in the water maze in CNQX-treated rats, a first hypothesis would be that the LHb is actively engaged in the modulaton of the HPA axis, as already envisaged in previous studies $[24,25]$, and that LHb inactivation would alter such a modulation. This however is rather unlikely. Anatomically there is no known direct projection from the LHb towards the hypothalamic paraventricular nucleus, so a direct modulation is unlikely. Nonetheless, as discussed by Jacinto et al. [25], the modulation of the HPA axis by the LHb could occur indirectly through its known regulatory control of key neurotransmitter systems, including serotonin [31], or noradrenalin [32,33]. Behaviourally, because there was no difference in CORT levels both in the elevated plus maze and in the home cage condition, one possibility is that, in the water maze, the higher CORT level in CNQX-treated rats is the consequence of memory deficits. These deficits could reflect the forgetting of the platform location or of the situation in general, so that the probe trial would be interpreted as a novel experience by CNQX-treated rats. Interestingly, in male Wistar rats Tabassum and Frey [34] showed that CORT levels in the water maze tend to be higher during a first swim experience than following a pre-training session, and Aguilar-Valles et al [35] showed that CORT levels decrease from day 1 to day 5 of the training phase in the water maze, suggesting that the response of the HPA axis adapts to the level of familiarity of a given situation. The fact that CNQX-treated rats exhibited higher levels of thigmotaxis during the probe trial suggests that the observed elevated CORT is the consequence of memory impairments. Thigmotaxis is the first behavioural manifestation observed during the initial acquisition period in the water maze [10,36,37]. Interestingly, MacGauran et al. [38] have found that introducing a conflict by rotating cues and start position during the probe trial induced impairments which were correlated with increased thigmotaxis. Therefore, it is likely that memory-impaired CNQX-treated rats, because they could not properly remember the location of the platform, automatically engaged in thigmotaxis during the probe trial. As a direct physiological consequence, the possible stress induced by the swim experience treated as novel is likely to have led to an increased CORT release ; this is consistent with the fact that naive rats have higher levels of blood CORT levels on a first swim day, in comparison to following training [34].

Finally, another possibility is that intra-LHb CNQX administration prevented rats to adequately respond to the stressful situation represented by the swim experience. Indeed, the LHb has recently been shown to play a key role in behavioural flexibility $[39,40]$; therefore, it is possible that the maintenance of a high level of thigmotaxis in CNQXtreated animals reflects the inability to switch to a more efficient search strategy. The hypothesis that the LHb is crucially involved in adapting motor strategies during stressful situations has already been proposed by Thornton and colleagues $[17,18]$. Huether et al $[41]$ proposed that stressors trigger a neuronal remodeling at the network level to generate coping patterns. Thus, during the water maze experiment, we could consider that after the elaboration of an appropriate behavior during training - the progressive amelioration of performances from day 1 to day 5 (see Supplementary data) -, during the probe trial LHb silencing disturbed the use of such a memory-based behavior and led rats to engage in an inappropriate strategy, i.e. thigmotaxis instead of a search of the platform at its location. Overall, it suggests that the LHb is involved in the generation and recall of adapted cognitive responses during emotionally challenging situations. The LHb could be considered as a pivotal brain structure required when a stressor goes from uncontrollable to controllable to generate adapted memory-based 
strategies. From an anatomical point of view, search strategies in the water maze are associated with the function of the basal ganglia, as for example lesions of the dorsomedial striatum in mice not only impairs memory performance but also markedly increase thigmotaxis [42]. Interestingly, Hong and Hikosaka [43] have demonstrated in monkeys that the LHb likely receives information from the whole basal ganglia, including the striatum, although indirectly. Therefore, one possibility is that the observed increased thigmotaxis in CNQX-treated animals is the consequence of altered communication between the striatum and the LHb. The resultant inability to adjust the behavioural response and initiate adequate search strategies, likely because of a defective modulation of dopamine transmission by the LHb, may contribute to the retrieval impairments observed in the probe trial.

In the present study we have found that blockade of excitatory inputs to the LHb induced memory deficits which were accompanied by an excessive release of CORT. Although a possible explanation is that this is the consequence of the perturbation of the potential direct role of the LHb in the modulation of the HPA axis, behavioural evidence rather suggest that altered CORT levels are the result of the memory deficits themselves, caused by impaired LHb function. Another possibility is that LHb dysfunction prevented rats from dealing with the stressful experience which the water maze represents, strengthening the view that the LHb is at the interface between emotional and cognitive processing. Therefore, increased thigmotaxis in CNQX-treated rats further suggests the LHb participates in behavioural adaptation relating to changes to the emotional aspects of the environment [44,45]. Finally, these findings also bring new insights to better understand the consequences of LHb dysfunction in pathologies such as depression, addiction and schizophrenia, among which hallmarks are cognitive deficits and altered emotional processing [46-50].

\section{Conflict of interest}

The authors declare that they have no conflict of interest.

\section{Acknowledgements}

This work was supported by the Centre National de la Recherche Scientifique, the University of Strasbourg, and the French government ( $\mathrm{PhD}$ fellowship to $\mathrm{VM}$ ). The authors wish to thank Dr. Monique Majchrzak for the gift of heparin-coated capillary tubes, and Wilf Gardner for critical reading of the manuscript.

\section{Appendix A. Supplementary data}

Supplementary data associated with this article can be found, in the online version, at https://doi.org/10.1016/j.bbr.2017.12.016.

\section{References}

[1] D.H. Root, C.A. Mejias-Aponte, J. Qi, M. Morales, Role of glutamatergic projections from ventral tegmental area to lateral habenula in aversive conditioning, J. Neurosci. Off. J. Soc. Neurosci. 34 (2014) 13906-13910.

[2] S.J. Shabel, C.D. Proulx, A. Trias, R.T. Murphy, R. Malinow, Input to the lateral habenula from the basal ganglia is excitatory, aversive, and suppressed by serotonin, Neuron 74 (2012) 475-481.

[3] A.M. Stamatakis, G.D. Stuber, Activation of lateral habenula inputs to the ventral midbrain promotes behavioral avoidance, Nat. Neurosci. 15 (2012) 1105-1107.

[4] M. Matsumoto, O. Hikosaka, Representation of negative motivational value in the primate lateral habenula, Nat. Neurosci. 12 (2009) 77-84.

[5] M. Agetsuma, H. Aizawa, T. Aoki, R. Nakayama, M. Takahoko, M. Goto, T. Sassa, R. Amo, T. Shiraki, K. Kawakami, T. Hosoya, S. Higashijima, H. Okamoto, The habenula is crucial for experience- dependent modification of fear responses in zebrafish, Nat. Neurosci. 13 (2010) 1354-1356.

[6] H. Okamoto, M. Agetsuma, H. Aizawa, Genetic dissection of the zebrafish habenula, a possible switching board for selection of behavioral strategy to cope with fear and anxiety, Dev. Neurobiol. 72 (2012) 386-394.

[7] M. Casarrubea, C. Davies, F. Faulisi, M. Pierucci, R. Colangeli, L. Partridge, S. Chambers, D. Cassar, M. Valentino, R. Muscat, A. Benigno, G. Crescimanno, G. Di Giovanni, Acute nicotine induces anxiety and disrupts temporal pattern organization of rat exploratory behavior in hole-board: a potential role for the lateral habenula, Front. Cell. Neurosci. 9 (2015) 197.

[8] V. Mathis, B. Cosquer, M. Avallone, J.-C. Cassel, L. Lecourtier, Excitatory transmission to the lateral habenula is critical for encoding and retrieval of spatial memory, Neuropsychopharmacology 40 (2015) 2843-2851.

[9] V. Mathis, A. Barbelivien, M. Majchrzak, C. Mathis, J.-C. Cassel, L. Lecourtier, The lateral habenula as a relay of cortical information to process working memory, Cereb. Cortex 27 (2017) 5485-5495.

[10] B.D. Devan, E.H. Goad, H.L. Petri, Dissociation of hippocampal and striatal contributions to spatial navigation in the water maze, Neurobiol. Learn. Mem. 66 (1996) 305-323.

[11] D. Treit, M. Fundytus, Thigmotaxis as a test for anxiolytic activity in rats, Pharmacol. Biochem. Behav. 31 (1988) 959-962.

[12] A.I. Herrero, C. Sandi, C. Venero, Individual differences in anxiety trait are related to spatial learning abilities and hippocampal expression of mineralocorticoid receptors, Neurobiol. Learn. Mem. 86 (2006) 150-159.

[13] S.A. Gagnon, A.D. Wagner, Acute stress and episodic memory retrieval: neurobiological mechanisms and behavioral consequences, Ann. N. Y. Acad. Sci. 1369 (2016) 55-75.

[14] N. Chastrette, D.W. Pfaff, R.B. Gibbs, Effects of daytime and nighttime stress on Foslike immunoreactivity in the paraventricular nucleus of the hypothalamus, the habenula, and the posterior paraventricular nucleus of the thalamus, Brain Res. 563 (1991) 339-344.

[15] W.E. Cullinan, J.P. Herman, D.F. Battaglia, H. Akil, S.J. Watson, Pattern and time course of immediate early gene expression in rat brain following acute stress, Neuroscience 64 (1995) 477-505.

[16] D. Wirtshafter, K.E. Asin, M.R. Pitzer, Dopamine agonists and stress produce different patterns of Fos- like immunoreactivity in the lateral habenula, Brain Res. 633 (1994) 21-26.

[17] E.W. Thornton, G.E. Bradbury, Effort and stress influence the effect of lesion of the habenula complex in one-way active avoidance learning, Physiol. Behav. 45 (1989) 929-935.

[18] E.W. Thornton, G.E. Bradbury, C. Davies, Increased immobility in an automated forced swimming test following lesion of the habenula in rats: absence of evidence for a contribution from motor impairment, Behav. Neurosci. 104 (1990) 37-43.

[19] A.F.T. Arnsten, Stress weakens prefrontal networks: molecular insults to higher cognition, Nat. Neurosci. 18 (2015) 1376-1385.

[20] B.S. McEwen, N.P. Bowles, J.D. Gray, M.N. Hill, R.G. Hunter, I.N. Karatsoreos, C. Nasca, Mechanisms of stress in the brain, Nat. Neurosci. 18 (2015) 1353-1363.

[21] J.P. Herman, H. Figueiredo, N.K. Mueller, Y. Ulrich-Lai, M.M. Ostrander, D.C. Choi, W.E. Cullinan, Central mechanisms of stress integration: hierarchical circuitry controlling hypothalamo-pituitary-adrenocortical responsiveness, Front. Neuroendocrinol. 24 (2003) 151-180.

[22] E.R. de Kloet, M.S. Oitzl, M. Joëls, Stress and cognition: are corticosteroids good or bad guys? Trends Neurosci. 22 (1999) 422-426.

[23] B.S. McEwen, R.M. Sapolsky, Stress and cognitive function, Curr. Opin. Neurobiol. 5 (1995) 205-216.

[24] C.A. Murphy, A.M. DiCamillo, F. Haun, M. Murray, Lesion of the habenular efferent pathway produces anxiety and locomotor hyperactivity in rats: a comparison of the effects of neonatal and adult lesions, Behav. Brain Res. 81 (1996) 43-52.

[25] L.R. Jacinto, R. Mata, A. Novais, F. Marques, N. Sousa, The habenula as a critical node in chronic stress-related anxiety, Exp. Neurol. 289 (2017) 46-54.

[26] H.C. Atkinson, B.J. Waddell, Circadian variation in basal plasma corticosterone and adrenocorticotropin in the rat: sexual dimorphism and changes across the estrous cycle, Endocrinology 138 (1997) 3842-3848.

[27] M. Fluttert, S. Dalm, M.S. Oitzl, A refined method for sequential blood sampling by tail incision in rats, Lab. Anim. 34 (2000) 372-378.

[28] C.L. Ferland, L.A. Schrader, Cage mate separation in pair-housed male rats evokes an acute stress corticosterone response, Neurosci. Lett. 489 (2011) 154-158.

[29] J.M. Koolhaas, A. Bartolomucci, B. Buwalda, S.F. de Boer, G. Flügge, S.M. Korte, P. Meerlo, R. Murison, B. Olivier, P. Palanza, G. Richter-Levin, A. Sgoifo, T. Steimer, O. Stiedl, G. van Dijk, M. Wöhr, E. Fuchs, Stress revisited: a critical evaluation of the stress concept, Neurosci. Biobehav. Rev. 35 (2011) 1291-1301.

[30] F. Spiga, J.J. Walker, J.R. Terry, S.L. Lightman, HPA axis-rhythms, Compr. Physiol. 4 (2014) 1273-1298.

[31] J. Amat, P.D. Sparks, P. Matus-Amat, J. Griggs, L.R. Watkins, S.F. Maier, The role of the habenular complex in the elevation of dorsal raphe nucleus serotonin and the changes in the behavioral responses produced by uncontrollable stress, Brain Res. 917 (2001) 118-126.

[32] M.A. Cenci, P. Kalén, R.J. Mandel, A. Björklund, Regional differences in the reg ulation of dopamine and noradrenaline release in medial frontal cortex, nucleus accumbens and caudate-putamen: a microdialysis study in the rat, Brain Res. 581 (1992) 217-228.

[33] P. Kalén, O.G. Nilsson, M.A. Cenci, E. Rosengren, O. Lindvall, A. Björklund, Intracerebral microdialysis as a tool to monitor transmitter release from grafted cholinergic and monoaminergic neurons, J. Neurosci. Methods 34 (1990) 107-115.

[34] H. Tabassum, J.U. Frey, The effect of acute swim stress and training in the water maze on hippocampal synaptic activity as well as plasticity in the dentate gyrus of freely moving rats: revisiting swim-induced LTP reinforcement, Hippocampus 23 (2013) 1291-1298.

[35] A. Aguilar-Valles, E. Sánchez, P. de Gortari, I. Balderas, V. Ramírez-Amaya, F. Bermúdez-Rattoni, P. Joseph-Bravo, Analysis of the stress response in rats trained in the water-maze: differential expression of corticotropin-releasing hormone, $\mathrm{CRH}$ R1, glucocorticoid receptors and brain-derived neurotrophic factor in limbic regions, Neuroendocrinology 82 (2005) 306-319.

[36] B.D. Devan, R.J. McDonald, N.M. White, Effects of medial and lateral caudate- 
putamen lesions on place- and cue-guided behaviors in the water maze: relation to thigmotaxis, Behav. Brain Res. 100 (1999) 5-14.

[37] B. Topic, E. Dere, D. Schulz, M.A. de Souza Silva, G. Jocham, E. Kart, J.P. Huston, Aged and adult rats compared in acquisition and extinction of escape from the water maze: focus on individual differences, Behav. Neurosci. 119 (2005) 127-144.

[38] A.M. McGauran, D. Harvey, L. Cunningham, S. Craig, S. Commins, Retention of cuebased associations in the water maze is time-dependent and sensitive to disruption by rotating the starting position, Behav. Brain Res. 151 (2004) 255-266.

[39] P.M. Baker, S.E. Oh, K.S. Kidder, S.J. Mizumori, Ongoing behavioral state information signaled in the lateral habenula guides choice flexibility in freely moving rats, Front. Behav. Neurosci. 9 (2015) 295.

[40] P.M. Baker, S.A. Raynor, N.T. Francis, S.J. Mizumori, Lateral habenula integration of proactive and retroactive information mediates behavioral flexibility, Neuroscience 345 (2017) 89-98.

[41] G. Huether, S. Doering, U. Rüger, E. Rüther, G. Schüssler, The stress-reaction process and the adaptive modification and reorganization of neuronal networks, Psychiatry Res. 87 (1999) 83-95.

[42] T. Pooters, I. Gantois, B. Vermaercke, R. D'Hooge, Inability to acquire spatial information and deploy spatial search strategies in mice with lesions in dorsomedial striatum, Behav. Brain Res. 298 (Pt B) (2016) 134-141.

[43] S. Hong, O. Hikosaka, Diverse sources of reward value signals in the basal ganglia nuclei transmitted to the lateral habenula in the monkey, Front. Hum. Neurosci. 7 (2013) 778.

[44] R. Amo, F. Fredes, M. Kinoshita, R. Aoki, H. Aizawa, M. Agetsuma, T. Aoki, T. Shiraki, H. Kakinuma, M. Matsuda, M. Yamazaki, M. Takahoko, T. Tsuboi, S. Higashijima, N. Miyasaka, T. Koide, Y. Yabuki, Y. Yoshihara, T. Fukai,

H. Okamoto, The habenulo-raphe serotonergic circuit encodes an aversive expectation value essential for adaptive active avoidance of danger, Neuron 84 (2014) 1034-1048.

[45] M. Stephenson-Jones, K. Yu, S. Ahrens, J.M. Tucciarone, A.N. van Huijstee, L.A. Mejia, M.A. Penzo, L.-H. Tai, L. Wilbrecht, B. Li, A basal ganglia circuit for evaluating action outcomes, Nature 539 (2016) 289-293.

[46] S. Cabib, S. Puglisi-Allegra, Stress, depression and the mesolimbic dopamine system, Psychopharmacology 128 (1996) 331-342.

[47] C. Corcoran, E. Walker, R. Huot, V. Mittal, K. Tessner, L. Kestler, D. Malaspina, The stress cascade and schizophrenia: etiology and onset, Schizophr. Bull. 29 (2003) $671-692$.

[48] F.V. Gomes, A.A. Grace, Adolescent stress as a driving factor for schizophrenia development-a basic science perspective, Schizophr. Bull. 43 (2017) 486-489.

[49] G.F. Koob, The darkness within: individual differences in stress, Cerebrum 4 (2015).

[50] A.M. Polter, J.A. Kauer, Stress and VTA synapses: implications for addiction and depression, Eur. J. Neurosci. 39 (2014) 1179-1188. 